\title{
Monitoring tuberculosis treatment outcome: analysis of national surveillance data from a clinical perspective
}

\author{
I C Ditah, ${ }^{1}$ M Reacher, ${ }^{2}$ C Palmer, ${ }^{1} \mathrm{~J}$ M Watson, ${ }^{3}$ J Innes, ${ }^{4}$ M E Kruijshaar, ${ }^{3}$ H N Luma, ${ }^{6}$ \\ I Abubakar ${ }^{3,5}$
}

\section{See Editorial, p 388}

${ }^{1}$ Institute of Public Health, Department of Public Health and Primary Care, University of Cambridge, Cambridge, UK; ${ }^{2}$ Regional Epidemiology Unit, Health Protection Agency, East of England, University Forvie Site, Cambridge, Cambridge, UK; ${ }^{3}$ Respiratory Diseases Department, Centre for Infections, Health Protection Agency, Colindale, London, UK;

${ }^{4}$ Birmingham Heartlands Hospital, Birmingham, UK;

${ }^{5}$ School of Medicine, Health

Policy and Practice, University of

East Anglia, Norwich, UK;

${ }^{6}$ Hospital Medicine, Hopital General Douala, Cameroon,

Faculty of Medicine and

Biomedical Sciences, Yaounde-

Cameroon

Correspondence to: Dr I Abubakar, Tuberculosis Section, Respiratory Diseases Department, Centre for Infections, Health Protection Agency, London NW9 5E0, UK; ibrahim.abubakar@hpa.org.uk

Received 23 October 2006 Accepted 22 June 2007 Published Online First 5 July 2007

\section{ABSTRACT}

Background: In 1998, the World Health Organization (WHO) and the International Union Against Tuberculosis and Lung Disease (IUATLD) published recommendations standardising the evaluation of tuberculosis treatment outcome in Europe. These guidelines fail to account for clinically appropriate alterations in the management of patients.

Objectives: To evaluate tuberculosis treatment outcome in England, Wales and Northern Ireland by redefining the outcome criteria and investigate factors associated with unsuccessful treatment outcome 12 months after notification.

Methods: This was a prospective analysis of a cohort of patients diagnosed in England, Wales and Northern Ireland and reported to the Enhanced Tuberculosis Surveillance system in 2001 and 2002. Proportions of success and failure were calculated based on a new set of criteria following discussion with clinicians treating tuberculosis cases. Logistic regression was used to study risk factors for unsuccessful treatment outcome.

Results: 13048 cases were notified in the study period. Of the 2676 that were identified as new sputum smear positive pulmonary cases, 2209 (82.5\%) had treatment outcome data reported. Using the WHO/IUATLD criteria, $76.8 \%$ were classified as successful. In contrast, applying the new criteria, the success rate was $87.5 \%$. This rate exceeds the $85 \%$ success target set by the WHO. Risk factors for unsuccessful treatment outcome included male sex (OR 1.27; 95\% Cl 1.08 to 1.49), being elderly ( $p$ trend $<0.001$ ), having pulmonary tuberculosis (OR 1.28; $95 \% \mathrm{Cl}$ 1.08 to 1.53$)$ and having resistance to any antituberculosis drug (OR 1.90; 95\% Cl 1.44 to 2.52).

Conclusion: The proportion of tuberculosis cases with a successful treatment outcome exceeded the target of $85 \%$ success rate based on the modified outcome categories. Although the tuberculosis treatment outcome criteria set by WHO/IUATLD appear to be clear, they mix measures of process and outcome. Further refinement may be necessary in low incidence high income countries, especially those with a high mortality among the elderly.

Tuberculosis is a leading cause of adult mortality arising from a single infectious agent globally. ${ }^{12}$ In England, Wales and Northern Ireland, the incidence of tuberculosis was 14.7 per 100000 population in 2005 and an estimated 400 patients die from tuberculosis yearly. ${ }^{3}$ In 1998, a working group of the World Health Organization (WHO) and the International Union Against Tuberculosis and Lung Disease (IUATLD) published recommendations standardising the surveillance of tuberculosis treatment outcome across Europe (table 1). ${ }^{45}$ These recommendations were based on applying standard short course treatment protocols to all new patients with sputum smear positive pulmonary tuberculosis. The WHO has also set targets for the detection and cure of at least $70 \%$ and $85 \%$, respectively.

A slightly modified version of the WHO/ IUATLD treatment outcome categories (table 2) that includes extrapulmonary tuberculosis is currently used in the UK. ${ }^{6}$ National surveillance data are collected through the Enhanced Tuberculosis Surveillance System (ETS) in England, Wales and Northern Ireland. As part of this system, treatment outcome monitoring was introduced in 2002.

Although the six mutually exclusive tuberculosis treatment outcome categories set by the WHO appear to be clear, it has been argued that they are primarily suitable for high incidence and low income countries. ${ }^{45}$ The definitions of treatment success and failure are not exhaustive and do not reflect decisions taken to account for individual case diagnostic results and therapeutic response. This is particularly relevant for low incidence industrialised countries with readily available resources, expertise and monitoring tools. For example, the outcome of a patient who has his or her treatment changed, suspended or prolonged by a physician for reasons such as adverse drug reactions or initial drug resistance are not considered satisfactory by the current recommendations. Therefore, the outcome definitions mix measures of process and outcome of patient care, for instance, by categorising treatment interruption, a process measure, as a final outcome. Such patients may eventually have a favourable outcome. Furthermore, the outcome category "death" has been the main reason for non-attainment of the $85 \%$ success target set by the WHO in many high income countries. ${ }^{7-9}$ In some of these deaths, tuberculosis is only incidental (other comorbidities present) and not causal. ${ }^{10}$ It is unreasonable to consider such cases as "failure". The analysis of death is further complicated by the inclusion of post mortem diagnosed cases in a system where autopsies are not undertaken in a systematic manner.

The aim of this study was to evaluate tuberculosis treatment outcome in England, Wales and Northern Ireland in 2001 and 2002 by redefining the criteria for "success" and "failure", from a clinical perspective. We have also investigated factors associated with an unfavourable tuberculosis treatment outcome. 
Table 1 Summary of tuberculosis treatment outcome criteria according to the WHO/IUATLD recommendations, including European guidelines

\begin{tabular}{|c|c|c|}
\hline \multicolumn{3}{|c|}{ Definite case with pulmonary tuberculosis } \\
\hline & Culture confirmed & Sputum smear microscopy confirmed \\
\hline Cured & $\begin{array}{l}\text { Documented conversion of culture during the } \\
\text { continuation phase }\end{array}$ & $\begin{array}{l}\text { Sputum smears negative on two occasions at } \\
\text { the end of treatment }\end{array}$ \\
\hline Treatment completed & $\begin{array}{l}\text { Documented treatment completion, but no } \\
\text { documented culture conversion }\end{array}$ & $\begin{array}{l}\text { Documented treatment completion, but not } \\
\text { sputum smear microscopy available at the } \\
\text { end of treatment }\end{array}$ \\
\hline Treatment failure & $\begin{array}{l}\text { Culture remaining or again becoming positive at } 5 \\
\text { months of treatment or later }\end{array}$ & $\begin{array}{l}\text { Sputum smears remaining or again becoming } \\
\text { positive at } 5 \text { months of treatment or later }\end{array}$ \\
\hline Death & $\begin{array}{l}\text { Death of the patient irrespective of cause at any time } \\
\text { before envisaged end of treatment }\end{array}$ & $\begin{array}{l}\text { Death of the patient irrespective of cause at } \\
\text { any time before envisaged end of treatment }\end{array}$ \\
\hline Treatment interrupted & $\begin{array}{l}\text { Patient off treatment for } 2 \text { consecutive months or } \\
\text { more or failure to complete treatment within } 9 \text { months } \\
\text { for a } 6 \text { month or within } 12 \text { months for a } 9 \text { month } \\
\text { regimen or drug intake less than } 80 \%\end{array}$ & $\begin{array}{l}\text { Patient off treatment for } 2 \text { consecutive } \\
\text { months or more or failure to complete } \\
\text { treatment within } 9 \text { months for a } 6 \text { month or } \\
\text { within } 12 \text { months for a } 9 \text { month regimen or } \\
\text { drug intake less than } 80 \%\end{array}$ \\
\hline Transfer out & $\begin{array}{l}\text { A patient referred to another clinician for treatment in } \\
\text { whom information on treatment outcome cannot be } \\
\text { obtained }\end{array}$ & $\begin{array}{l}\text { A patient referred to another clinician for } \\
\text { treatment in whom information on treatment } \\
\text { outcome cannot be obtained }\end{array}$ \\
\hline
\end{tabular}

Successful treatment outcome: patients declared cured or completed treatment.

Unsatisfactory outcome: patients who interrupted treatment, were transferred out or failed on treatment,

Death, patients who died before the diagnosis of tuberculosis was established or died during the course of treatment either with or from tuberculosis.

IUATLD, International Union Against Tuberculosis and Lung Disease; WHO, World Health Organization.

\section{METHODS}

This was a prospective follow-up of a cohort of tuberculosis patients diagnosed in England, Wales and Northern Ireland and reported to the ETS system in the calendar years 2001 and 2002. At diagnosis, a standard form which permits the collection of clinical and demographic data on age, sex, residence, ethnicity, place of birth, date of diagnosis, disease site, sputum smear and culture status (where available) is completed by the clinician.
Typically, tuberculosis treatment lasts 6 months. Less commonly, it may last up to 12 months for tuberculosis meningitis or longer among patients with rifampicin or multi-drug resistant disease. ${ }^{11}$ The treatment outcome form reports on the status of the patient 12 months after notification, regardless of whether treatment has been completed. When treatment outcome monitoring forms were not returned, local coordinators contacted clinicians to improve the completeness of data.

Table 2 Definitions of tuberculosis treatment outcome indicators in England, Wales and Northern Ireland

\begin{tabular}{ll}
\hline Indicator & Definition \\
\hline Cure & $\begin{array}{l}\text { Completed full course of antituberculosis chemotherapy within } 12 \text { months of starting } \\
\text { treatment or notification and documented culture conversion in sputum positive patients } \\
\text { during treatment (ie, culture positive at start of treatment becoming culture negative) } \\
\text { Completed full course of therapy within } 1 \text { year of starting treatment/notification }\end{array}$ \\
Treatment stopped & $\begin{array}{l}\text { Patient found to have stopped treatment (by himself) or any other reason not mentioned } \\
\text { below } \\
\text { Death }\end{array}$ \\
& $\begin{array}{l}\text { Patients who die while on treatment for tuberculosis or who were diagnosed post mortem or } \\
\text { died without starting treatment }\end{array}$
\end{tabular}

Still on treatment at 1 year: patient who is still on treatment at 1 year because:

(a) Initially planned course $\quad$ Regimen of 12 months or longer was planned at start of treatment (eg, CNS tuberculosis or

(b) Interruption drug resistant disease)

Non-completion of a treatment regimen initially planned to last 12 months or less (but still taking initially planned combination of drugs) as a result of:

interruption due to intolerance/side effects

drug intake less than $80 \%$ of prescribed dose

other interruption in taking prescribed treatment for 2 months or longer

(c) Change

A change and/or extension of the drug regimen as a result of (select one or more): intolerance/side effects initial drug resistance development of new drug resistance (change in drug susceptibilities during current treatment episode)

failure to culture convert

poor clinical response to treatment

Lost to follow-up

Transferred out

Unknown
Patient lost to follow-up before the end of treatment

Responsibility for patient's care transferred to another clinical team

No treatment details available (eg, lost patient notes) 
Table 3 Summary of the new criteria used to define successful and unsuccessful tuberculosis treatment outcome in England, Wales and Northern Ireland

\begin{tabular}{lll}
\hline \multirow{2}{*}{ UK outcome categories } & \multicolumn{2}{l}{ Modified criteria for monitoring clinical outcome } \\
\cline { 2 - 3 } $\begin{array}{ll}\text { Completed treatment } \\
\text { Died }\end{array}$ & Successful & Unsuccessful \\
& Tuberculosis incidental to death & Tuberculosis caused death, contributed to \\
& & $\begin{array}{l}\text { death or relationship between the two } \\
\text { unknown }\end{array}$ \\
Still on treatment & $\begin{array}{l}\text { Still on initially planned course of treatment; } \\
\text { had treatment changed or extended as a result } \\
\text { of drug intolerance, adverse side effects or } \\
\text { initial drug resistance; failure to culture or } \\
\text { smear convert from positive to negative; and }\end{array}$ & $\begin{array}{l}\text { Any other reason mentioned in table 2 as } \\
\text { reason for still being on treatment, but not } \\
\text { poor clinical response on treatment. }\end{array}$ \\
\cline { 2 - 3 } Lost to follow-up & - & All the cases under successful outcome. \\
Stopped treatment & - & $\begin{array}{l}\text { All cases who interrupted treatment with no } \\
\text { justifiable reason. }\end{array}$ \\
Unknown & - & All (treatment details not available)
\end{tabular}

All cases that left the country before the treatment completion point were considered to have a "neutral" outcome and were dropped from the analysis.

Information on Mycobacterium tuberculosis complex isolates and drug sensitivity profile is collated through the UK Mycobacterial Surveillance Network (MycobNet). At the national level, both databases are checked in a multistep process. Firstly, the data are checked for duplicates. The information is then fed back to local ETS coordinators for verification before returning the confirmed data to the national level. The two databases are subsequently linked using an inhouse matching software to produce pairs of possible matches based on name, sex, residential address and date of birth. Pairs with a very high degree of similarity are automatically matched. Further pairs with a high matching score are reviewed individually.

\section{Definition of terms}

In England, Wales and Northern Ireland, all (pulmonary and extrapulmonary) cases of tuberculosis are notifiable. ${ }^{6}$ A case can either be culture confirmed (definite) or other than culture confirmed (a clinician's judgment based on clinical features with or without radiological, histological or tuberculin skin test evidence of tuberculosis, and the decision to treat a patient with a full course of antituberculosis medication). Pulmonary tuberculosis refers to tuberculosis disease involving the lung parenchyma with or without extrapulmonary disease, while extrapulmonary tuberculosis can involve any organ other than the lungs. A cohort is considered as the group of patients notified within one calendar year and whose treatment outcome is to be reported. For this work, analysis included all cases notified in 2001 and 2002. "Any drug resistance" refers to resistance to any firstline antituberculosis drug, while "multidrug resistance" is resistance to at least isoniazid and rifampicin. Table 2 summarises the treatment outcome definitions used in England, Wales and Northern Ireland.

\section{Analysis of tuberculosis treatment outcome}

The outcome of the treatment of each case was reclassified as either "success" or "failure". The criteria used are outlined in table 3. Cases diagnosed post mortem were dropped from the analytic cohort.

The current UK criteria were used to describe the outcome of all cases in a flow chart (fig 1).

To calculate the proportion of cases with a successful outcome, three scenarios were used. Firstly, the proportions of treatment outcome were calculated based on the WHO/ IUATLD recommendations (table 1) (ie, including only cases with sputum smear positive pulmonary disease). Secondly, the new criteria (table 3) were applied to this same group of patients. Finally, the new criteria were used to determine the proportion with a successful outcome among all patients with tuberculosis (pulmonary and extrapulmonary).

\section{Risk factor analysis}

To determine the risk factors for unfavourable treatment outcome, the entire cohort, including extrapulmonary cases, was used to increase our statistical resolution. By single variable logistic regression analysis, the relationship between the outcome of tuberculosis treatment and case characteristics was examined. The characteristics examined included age, gender, ethnicity, place of birth, reporting region, site of disease, previous history of tuberculosis disease, and bacteriological and drug sensitivity test results. The odds ratio (95\% confidence interval (CI)) was used to assess the strength of associations. The Pearson $\chi^{2}$ test with a two tailed significance limit set at 0.05 was used to evaluate the role of chance. For the ordinal variable age, the $\chi^{2}$ test for trend was used. In a multivariable logistic regression model, we adjusted for all variables that attained statistical significance in the univariable analysis. The likelihood ratio test was used to test for the interaction between the different risk factor variables. All analyses were done using Intercooled Stata 9, Stata Corporation Texas, USA.

\section{RESULTS}

\section{Study population}

A total of 13048 cases were reported in the study period. Median age of all cases was 36 years (interquartile range $26-57$ ). A majority were male (54.0\%). The largest proportion (35.3\%) of patients reported were from the Indian, Pakistani and Bangladeshi ethnic groups: $26.2 \%$ of cases were white Caucasians, $18.2 \%$ black Africans and $2.9 \%$ black Caribbeans. The Chinese ethnic group were the least represented (1.4\%). Fifty-seven per cent (7497) were born abroad and $42.7 \%$ of cases were reported from London. Most cases (58.0\%) had pulmonary tuberculosis. Among cases of white ethnicity (3420), the proportion with pulmonary disease was $76.7 \%$ (2624). Exploratory analysis showed that patients with and without 
Figure 1 Summary of tuberculosis (TB) treatment outcome of cases reported in England, Wales and Northern Ireland in 2001 and 2002.

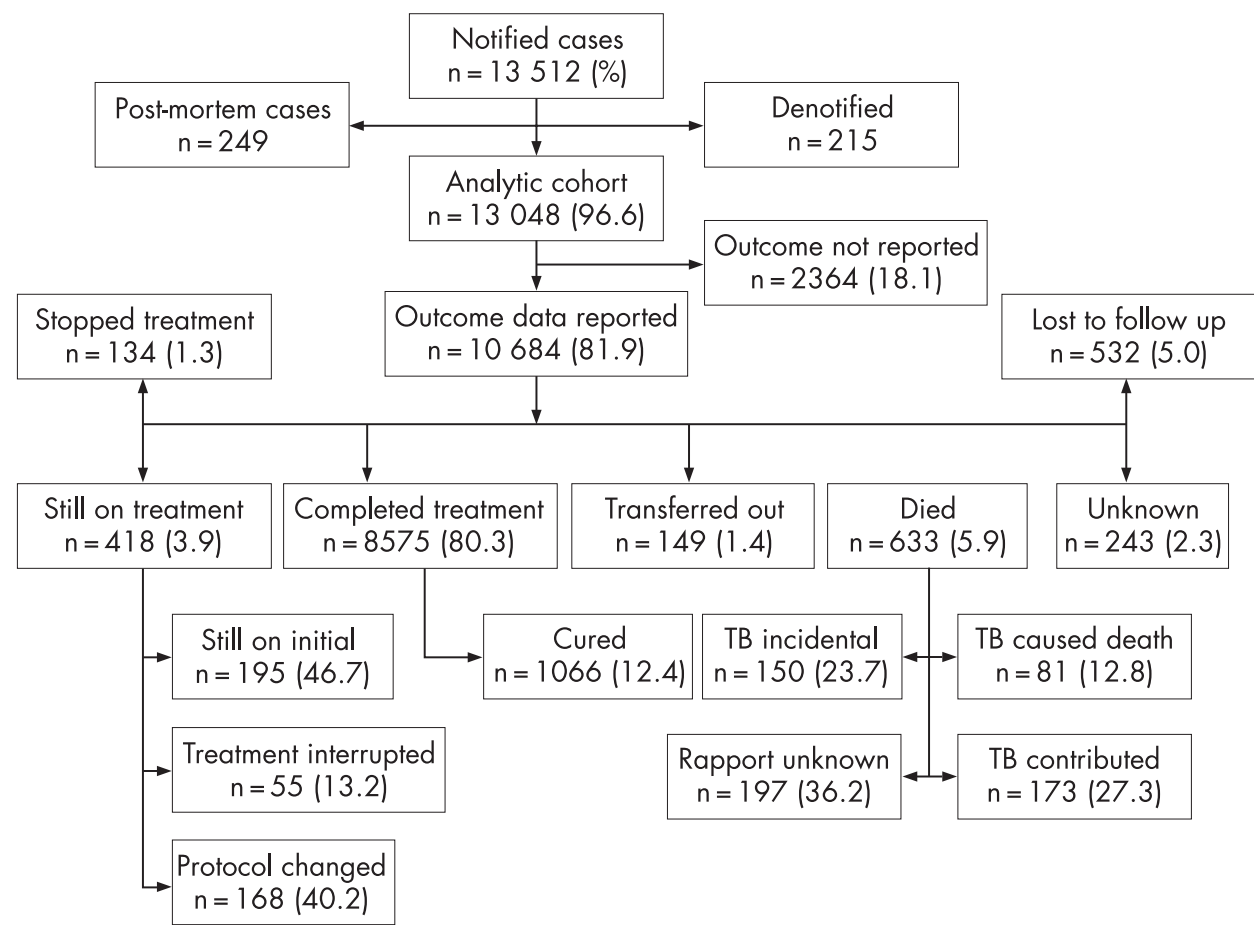

an outcome reported differed significantly by ethnicity $(p<0.001)$, disease site $(p=0.05)$ and age $(p<0.001)$.

\section{Tuberculosis treatment outcome}

Of the 13048 subjects included, treatment outcome data were reported on 10684 cases. Figure 1 summarises the treatment outcome results according to the outcome categories used by the UK national surveillance programme: $18.1 \%$ did not have outcome data reported. Regional variation in the number of cases notified and the proportion with outcome data reported are shown in table 4.

According to the WHO/IUATLD recommendations, 2676 cases were eligible for inclusion (ie, new sputum smear positive cases). Of these, 2209 (82.5\%) had outcome data on treatment reported. Among the 1696 (76.8\%) cases who completed treatment, 500 (29.5\%) were declared cured. Table 5 summarises the treatment success and failure proportions according to the WHO/IUATLD and new (modified) criteria.

Table 4 Number of tuberculosis cases notified with treatment outcome reported in England, Wales and Northern Ireland in 2001 and 2002 by UK region

\begin{tabular}{lcr}
\hline Region & No notified & $\begin{array}{c}\text { No with outcome } \\
\text { reported (\%) }\end{array}$ \\
\hline East Midland & 1015 & $405(40.0)$ \\
East England & 654 & $565(86.4)$ \\
London & 5578 & $5120(91.8)$ \\
North East & 310 & $274(88.4)$ \\
North West & 1243 & $901(72.5)$ \\
Northern Ireland & 119 & $111(93.3)$ \\
South East & 895 & $432(48.3)$ \\
South West & 417 & $382(91.6)$ \\
Wales & 318 & $275(86.5)$ \\
West Midlands & 1457 & $1232(84.6)$ \\
Yorkshire and the Humber & 1042 & $987(94.7)$ \\
Total & 13048 & $10684(81.9)$ \\
\hline
\end{tabular}

Determinants of tuberculosis treatment outcome in England, Wales and Northern Ireland in 2001 and 2002

Table 6 shows the univariable (unadjusted) and multivariable (adjusted) OR, 95\% CI and p values for the association between patient characteristics and the outcome of tuberculosis treatment (failure or success) in England, Wales and Northern Ireland. In the unadjusted analysis, factors that were significantly associated with unsuccessful treatment outcome included male sex (OR 1.37; 95\% CI 1.22 to 1.54), aged 15 years and over (OR 1.48, 95\% CI 1.34 to 1.65; p value for trend $<0.001$ ), being reported in London (OR 1.47; 95\% CI 1.31 to 1.64), having pulmonary tuberculosis (OR 1.48; 95\% CI 1.31 to 1.66) and having disease resistant to any drug (OR 1.69; $95 \% \mathrm{CI}$ 1.31 to 2.18$)$. MDR tuberculosis had a borderline statistical association (OR 2.09; 95\% CI 1.02 to 4.28) with unsuccessful interval. The ethnic groups black Caribbean (OR 0.66; 95\% CI 0.56 to 0.77 ), India/Pakistani/Bangladeshi (OR 0.77; 95\% CI 0.65 to 0.92 ) and any other (OR $0.52 ; 95 \%$ CI 0.45 to 0.60 ) compared with the white ethnic group were significantly associated with a favourable treatment outcome.

Adjusting for age, sex, place of birth, ethnic group, disease site, region and resistance to at least one firstline drug, factors that were still statistically associated with unfavourable outcome included: male sex (OR $1.27 ; 95 \%$ CI 1.08 to 1.49 ), age

Table 5 Tuberculosis treatment success and failure rates according to

\begin{tabular}{llll}
\hline \multirow{2}{*}{ Category } & \multicolumn{3}{l}{ Outcome criteria used: } \\
\cline { 2 - 4 } & WHO/IUATLD* & New (modified)* & New (modified) $\uparrow$ \\
\hline Success (\%) & 76.8 & 87.5 & 86.6 \\
Unsatisfactory (\%) & 23.2 & 12.5 & 13.4
\end{tabular}

*Only new sputum smear positive pulmonary (2676) tuberculosis cases were included.

†The entire cohort (ie, including both pulmonary and extrapulmonary tuberculosis cases (13 048)).

IUATLD, International Union Against Tuberculosis and Lung Disease; WHO, World Health Organization. treatment outcome, although with a relatively wide confidence the WHO and new criteria 
Table 6 Determinants of unsuccessful tuberculosis treatment outcome in England, Wales and Northern Ireland of cases reported in 2001 and 2002

\begin{tabular}{|c|c|c|c|c|c|c|}
\hline \multirow[b]{2}{*}{ Risk factor } & \multicolumn{2}{|c|}{ Treatment outcome } & \multicolumn{2}{|c|}{$\begin{array}{l}\text { Univariable analysis for } \\
\text { failure }\end{array}$} & \multicolumn{2}{|c|}{$\begin{array}{l}\text { Multivariable analysis for } \\
\text { failure }\end{array}$} \\
\hline & $\begin{array}{l}\text { Failure } \\
(n=1412)\end{array}$ & $\begin{array}{l}\text { Success } \\
(\mathrm{n}=9123)\end{array}$ & OR (95\% Cl) & p Value & ORa (95\% CI) & p Value \\
\hline Sex & & & 1 & & 1 & 0.003 \\
\hline Female & 551 & 4262 & $1.37(1.22,1.54)$ & $<0.001$ & $1.27(1.08-1.49)$ & \\
\hline Male & 859 & 4848 & & & & \\
\hline Age (y) & & & $\mathrm{p}$ trend & $<0.001$ & $\mathrm{p}$ trend & $<0.001$ \\
\hline$<15$ & 48 & 622 & 1 & & $1.93(0.92,4.06)$ & \\
\hline $15-44$ & 678 & 5311 & $1.65(1.22,2.24)$ & & $2.29(1.07,4.9)$ & \\
\hline $45-64$ & 275 & 1838 & $1.94(1.41,2.67)$ & & $4.33(2.02,9.25)$ & \\
\hline $65+$ & 411 & 1350 & $3.95(2.88,5.40)$ & & & \\
\hline \multicolumn{7}{|l|}{ Place of birth } \\
\hline UK & 469 & 2724 & 1 & & 1 & \\
\hline Abroad & 762 & 5672 & $0.78(0.69,0.88)$ & $<0.001$ & $1.07(0.82,1.38)$ & 0.629 \\
\hline Unknown & 181 & 727 & $1.45(1.2,1.75)$ & $<0.001$ & $1.60(1.19,2.15)$ & 0.002 \\
\hline \multicolumn{7}{|l|}{ Ethnic group } \\
\hline White Caucasian & 496 & 2277 & 1 & & 1 & \\
\hline Black African & 51 & 284 & $0.83(0.60,1.13)$ & 0.227 & $1.17(0.76,1.81)$ & 0.47 \\
\hline Black Caribbean & 255 & 1780 & $0.66(0.56,0.77)$ & $<0.001$ & $1.24(0.90,1.72)$ & 0.194 \\
\hline India/Pakistani/Bangladesh & 208 & 1234 & $0.77(0.65,0.92)$ & 0.004 & $1.16(0.83,1.61)$ & 0.38 \\
\hline Other & 402 & 3548 & $0.52(0.45,0.60)$ & $<0.001$ & $0.70(0.56,0.79)$ & 0.01 \\
\hline \multicolumn{7}{|l|}{ Site of disease } \\
\hline EPTB & 468 & 3846 & 1 & & 1 & \\
\hline PTB & 922 & 5156 & $1.48(1.31,1.66)$ & $<0.001$ & $1.28(1.08,1.53)$ & 0.006 \\
\hline \multicolumn{7}{|l|}{ Region } \\
\hline Out of London & 559 & 4472 & 1 & & $1.48(1.31,1.68)$ & $<0.001$ \\
\hline London & 853 & 4651 & $1.47(1.31,1.64)$ & $<0.001$ & & \\
\hline \multicolumn{7}{|l|}{ History of previous $\mathrm{Rx}$} \\
\hline No & 1340 & 8786 & 1 & & & \\
\hline Yes & 55 & 283 & $1.27(0.95,1.71)$ & 0.107 & & \\
\hline \multicolumn{7}{|l|}{ Culture result } \\
\hline Negative & 583 & 3709 & 1 & & & \\
\hline Positive & 829 & 5414 & $0.97(0.87,1.09)$ & 0.652 & & \\
\hline \multicolumn{7}{|l|}{ Any drug resistance } \\
\hline No & 683 & 4597 & 1 & & 1 & \\
\hline Yes & 81 & 323 & $1.69(1.31,2.18)$ & $<0.001$ & $1.90(1.44,2.52)$ & $<0.001$ \\
\hline \multicolumn{7}{|l|}{ MDR } \\
\hline No & 754 & 4889 & 1 & & 1 & \\
\hline Yes & 10 & 31 & $2.09(1.02,4.28)$ & 0.044 & $1.21(0.56,2.61)$ & 0.635 \\
\hline
\end{tabular}

The model for the multivariable analysis included all variables that were significantly associated with treatment outcome in the single variable analysis.

EPTB, extrapulmonary tuberculosis; MDR, multi-drug resistance; $\mathrm{OR}$, odds ratio; $\mathrm{ORa}$, adjusted odds ratio; PTB, pulmonary tuberculosis; Rx, treatment

15 years and over (OR 1.48; 95\% CI 1.34 to 1.65 , p value for trend $<0.001$ ), people with unknown place of birth (OR 1.60; $95 \%$ CI 1.19 to 2.15 ), pulmonary tuberculosis (OR $1.28 ; 95 \%$ CI 1.08 to 1.53 ) and having resistance to any antituberculosis medication (OR 1.90; 95\% CI 1.44 to 2.52).

\section{DISCUSSION}

This study shows that the treatment outcome of sputum smear positive pulmonary tuberculosis cases was successful in $87.5 \%$ using the new criteria in England, Wales and Northern Ireland in 2001 and 2002. This success rate exceeds the WHO target rate of $85 \%$, in contrast with the $76.8 \%$ rate obtained using the WHO treatment outcome surveillance criteria. The study also suggests that the main factors associated with unfavourable tuberculosis treatment outcome include male sex, higher age, people with unknown place of birth, having pulmonary tuberculosis or resistance to any tuberculosis medication.
Tuberculosis outcome surveillance is important for two reasons. Firstly, it allows the measurement and comparison of the performance of tuberculosis services locally, regionally, nationally and internationally. The collection of information on outcomes depends heavily on the collaboration of local tuberculosis departments. The departments will do this better if the exercise is beneficial to them. This is the second important reason for tuberculosis treatment outcome surveillance. It allows local tuberculosis departments to list all cases where the tuberculosis service failed, and perform a review of such cases. Even a large department in the UK will have no more than about 20 such cases in a year. There may be much to be learned from discussion of the details of these cases in regular meetings by tuberculosis case managers and other responsible partners.

The main difficulty in converting the WHO treatment outcome definitions into categories of success and failure in a high income low incidence context such as the UK is what to do 
with those patients who are still on treatment after 12 months, those transferred out and patients who have either died or whose treatment is interrupted for clinical reasons. To clinicians managing tuberculosis, treatment for longer than 12 months is much less of a problem. Most have had their initial treatment plan appropriately adjusted in the face of toxicity or drug resistance and can be predicted after 12 months to do well. They should then be classed as successes. A few remain a cause for concern as they may still be culture positive at this stage. These should be classed as failures. Patients with rifampicin or multi-drug resistant disease, in addition, should have their final outcome monitored at 24 and 36 months. Perhaps a modification to the treatment outcome reporting form would allow these distinctions to be made.

It was not possible to classify the majority of patients who completed treatment as cured by virtue of a negative follow-up culture. Ideally, it would be useful to assess outcome based on a cohort of culture positive and culture converted cases. However, only a small proportion of cases (29.5\%) had culture conversion documented. This is probably one of the reasons why the WHO recommends that cases declared cured or who complete treatment be considered a "success" and analysed as one group. Nevertheless, it should be standard practice to obtain follow-up cultures after 2 months and at completion of treatment in tuberculosis patients wherever possible. Usually difficulties in obtaining samples from patients who are no longer symptomatic hamper the bacteriological confirmation of cure. Invasive methods such as induced sputum, gastric aspiration and bronchoscopy may not be appropriate for monitoring in all such cases. However, such investigations may have high public health significance in pulmonary cases, as this would not only ascertain infectiousness but also permit the identification of cases who require close clinical and therapeutic monitoring.

As suggested by previous authors, ${ }^{6}{ }^{12-14}$ we excluded post mortem diagnosed cases. Including these cases leads to an overestimation of the number of tuberculosis cases because of the difficulty in distinguishing inactive from active tuberculosis disease on necropsy specimens. ${ }^{15}$ In addition, most of the elderly who account for a large proportion of the deaths may die with and not from tuberculosis. Moreover, post mortem examinations are not consistently carried out in the UK. This inconsistency makes within and between country comparisons inappropriate. This study also used treatment cohorts for 2 years, contrary to the WHO recommended 1 yearly cohort analysis. This increased our statistical resolution in studying risk factors for unsuccessful treatment outcome. Over the 2 year period, there was no major change in the case definition, management and surveillance guidelines for tuberculosis in the UK. The cohorts were therefore comparable.

These results should be interpreted bearing in mind the following weaknesses. The information used for the analysis was derived from routine data with potential for errors in coding, and between observer variability that are inherent in the collection and entry. Incomplete or inaccurate matching between the different surveillance databases (MycobNet and ETS) is possible. This could lead to the observation of spurious and/or null associations. However, the routine application of rigorous checks, including automated and manual checks in the ETS system, reduce the probability of these errors. Differences in the extent to which clinicians ascertain outcome in pulmonary and extrapulmonary cases may be another limiting factor contributing to differences in outcome.
Failure to report outcome data in about one-fifth of the sputum smear positive pulmonary tuberculosis cases is disappointing given its public health implications. The main shortcoming of the first 2 years following the introduction of tuberculosis treatment outcome monitoring as a component of the ETS was implementation and logistic difficulties in some regions. Two UK regions had particularly low outcome report results. However, there is evidence of improvement in case outcome reports from initial analysis of subsequent national tuberculosis outcome surveillance data from these regions. ${ }^{3}$ Perhaps timely dissemination of outcome reports to the different partners involved in the data collection process could serve as a motivation to trigger more rigorous case finding and complete reporting. A pilot system for active follow-up of missing cases has been set up and should inform future data.

In addition, the group without outcome data were more likely to be white Caucasians, have pulmonary disease and be older, all factors associated with unfavourable treatment outcome in this study. This implies that the outcome of the entire cohort is likely to be less than the observed success rate.

The denotification rate in this study appeared small. This may be explained by the delay between statutory notification and completion of ETS forms. In many parts of the country, cases initially reported to the ETS and later found not to have tuberculosis are denotified early in the process before the data are forwarded to the national level.

In our risk factor analysis, those with an unknown place of birth were found to have a higher risk of unsuccessful treatment outcome, which may be a proxy for other unmeasured indicators of poor outcome. Statistical adjustment was limited only to the variables routinely collected by the tuberculosis surveillance system. Comorbidity and risk factors such as alcohol dependence, homelessness, imprisonment, injecting drug use, immigration status, unemployment and HIV infection (as demonstrated by some other studies) could explain at least in part some of the association observed in our regression model. ${ }^{16} 17$ The findings of this study, however, agree with previous reports that adjusted for some of these factors. ${ }^{7}$

The above findings have both public health and clinical implications. Our results strengthen and build upon earlier criticisms that the WHO tuberculosis treatment outcome criteria are primarily suitable for high burden and low income countries. ${ }^{4-6}$ It has also been argued that they mix measures of process and outcome in a way that makes the results of tuberculosis treatment outcome difficult to interpret from a clinical perspective. The targets for treatment currently suggested by the WHO are not attainable in settings with very high mortality rates among elderly patients who may die with, rather than of, tuberculosis. ${ }^{10} 1819$ In addition, variation in clinical management and the use of modified standardised courses of therapy that result in eventual cure are more likely in a resource rich setting. The modified criteria used in this study give a better insight into the effectiveness of tuberculosis treatment services in case holding and ability to complete treatment. These evaluation criteria could be considered by the WHO for low incidence and resource rich countries.

In conclusion, although the tuberculosis treatment outcome criteria set by the WHO appear to be clear and comprehensive, they have limitations and require further refinement in well resourced countries to permit an objective evaluation of tuberculosis treatment programmes. Tuberculosis management should integrate risk assessment for unsuccessful treatment outcome. There is a need for the collection of information on comorbid states and detailed cause of death associated with 
tuberculosis by surveillance systems in order to give a better understanding of treatment outcome. Future research should focus on identifying causes of treatment failure, including default from treatment and mortality (a common reason for unsuccessful treatment outcome) in tuberculosis patients, especially among the elderly.

Acknowledgements: We wish to acknowledge the contribution of all tuberculosis nurses and clinicians who report cases to the enhanced tuberculosis surveillance system. We are grateful to the local and regional surveillance coordinators for collating the data and to David Quinn and Jonathan Crofts for their work in maintaining the enhanced surveillance database. We wish to thank Dr Jeremiah Ngondi for comments on drafts of the manuscript.

Competing interests: None.

Ethics approval: This study was carried out with national surveillance data. The Health Protection Agency has PIAG approval to hold and analyse national surveillance data for public health purposes.

\section{REFERENCES}

1. World Health Organization. Global tuberculosis control: Surveillance, planning and financing. WHO report. WHO/HTM/TB/2006.362. Geneva: WHO, 2006.

2. World Health Organization. Treatment of tuberculosis: Guidelines for National Programmes, 3rd Edn. WHO/CDC/TB/2003.313. Geneva: WHO, 2003

3. Tuberculosis Section. Focus on Tuberculosis: 2006 Surveillance Report, England Wales and Northern Ireland. London: Health Protection Agency, 2006.

4. Veen J, Raviglione M, Rieder $\mathrm{HL}$, et al. Standardized tuberculosis treatment outcome monitoring in Europe. Recommendations of a Working Group of the World Health Organization (WHO) and the European Region of the International Union Against Tuberculosis and Lung Disease (IUATLD) for uniform reporting by cohort analysis of treatment outcome in tuberculosis patients. Eur Respir J 1998;12:505-10.

5. Broekmans JF, Migliori GB, Rieder HL, et al. European framework for tuberculosis control and elimination in countries with a low incidence. Recommendations of the World Health Organization (WHO), International Union Against Tuberculosis and Lung
Disease (IUATLD) and Royal Netherlands Tuberculosis Association (KNCV) Working Group. Eur Respir J 2002;19:765-75.

6. Tuberculosis Section Respiratory Diseases Department Communicable Disease Surveillance Centre Health Protection Agency. First Annual Report on Tuberculosis Treatment Outcome Surveillance in England, Wales and Northern Ireland: Outcome Results on Tuberculosis Cases Reported in 2001. London: Tuberculosis Section Respiratory Diseases Department Communicable Disease Surveillance Centre Health Protection Agency, 2004.

7. Farah MG, Tverdal A, Steen TW, et al. Treatment outcome of new culture positive pulmonary tuberculosis in Norway. BMC Public Health 2005;5:14.

8. Faustini A, Hall AJ, Perucci CA. Tuberculosis treatment outcomes in Europe: a systematic review. Eur Respir J 2005;26:503-10.

9. Diel R, Niemann S. Outcome of tuberculosis treatment in Hamburg: a survey, 19972001. Int J Tuberc Lung Dis 2003;7:124-31.

10. Ormerod LP, Prescott RJ. The management of pulmonary and lymph node tuberculosis notified in England and Wales in 1998. Clin Med 2003;3:57-61.

11. National Collaborating Centre for Chronic Conditions. Tuberculosis: clinical diagnosis and management of tuberculosis, and measures for its prevention and control. London: Royal College of Physicians, 2006.

12. Krejbich F, Trnka L, Mazankova V. Tuberculosis in the Czech Republic in 2002. Cas Lek Cesk 2003;142:746-50.

13. Trnka L, Dankova D, Krejbich F. Is quarterly cohort analysis useful for assessing treatment outcomes in a low incidence country? Int J Tuberc Lung Dis 2001:5:250-6.

14. Lillebaek T, Poulsen S, Kok-Jensen A. Tuberculosis treatment in Denmark: treatment outcome for all Danish patients in 1992. Int J Tuberc Lung Dis 1999;3:603-12.

15. Chapman RC, Claydon SM. Mycobacterium tuberculosis: a continuing cause of sudden and unexpected death in west London. J Clin Pathol 1992;45:713-15.

16. Antoine D, Maguire H, Story A. Epidemiology and response to the growing problem of tuberculosis in London. Euro Surveill 2006;11:25-8.

17. Terris M. Relation of economic status to tuberculosis mortality by age and sex Am J Public Health 1948;38:1061-70.

18. Fielder JF, Chaulk CP, Dalvi M, et al. A high tuberculosis case-fatality rate in a setting of effective tuberculosis control: implications for acceptable treatment success rates. Int J Tuberc Lung Dis 2002;6:1114-17.

19. Conaty SJ, Dart S, Hayward AC, et al. Reasons for low reported treatment success in notified tuberculosis patients from a London hospital according to new outcome reporting. Commun Dis Public Health 2004;7:73-6. 